\title{
TOPOLOGICAL GROUP FOUNDATIONS OF RIGID SPACE GEOMETRY
}

\author{
BY \\ DEANE MONTGOMERY AND LEO ZIPPIN
}

Dedicated to the memory of Bella Zippin, mother of one and friend of both of us.

1. Hilbert, af ter building up geometry from a point of view which relegated continuity considerations to the background [4], built up plane geometry afresh [5] on the foundation of groups of homeomorphisms of the number plane, both "continuity" concepts. It is this latter point of view with which we are concerned in this paper. Hilbert carried out this program only for the plane but he hinted that it might be possible to carry it out in a somewhat similar way for three-space. Kerékjártó took up the problem [6] for threespace and made a great deal of progress with it, but, as he wrote before the recent developments in topological groups, he found it necessary to employ a stronger set of axioms than is necessary now.

Relying on the theory of topological groups we recently characterized the rotation group of three-space [9], and in commenting on that work P. A. Smith [12] suggested that it might provide the basis for an extension of Hilbert's program to three-space.

The purpose of this paper is twofold. In the first place we shall characterize the classical space geometries on the basis of a fairly weak set of axioms, and in the second place we shall show that Hilbert's axioms for the case of the plane can be weakened by replacing what might be called his "three-point condition" by a two-point condition. We achieve this latter purpose more or less incidentally to the first.

In comparing our set of three-space axioms with Hilbert's axioms for the plane we find that the first axiom is the same in both cases. The third axiom of this paper is weaker than Hilbert's, and our second purpose above is to show that this weaker axiom also suffices in Hilbert's case. Our second axiom is weaker than Hilbert's second axiom in that it relates to the subgroup leaving a single point fixed, but it is incomparably stronger in what it asks of that one subgroup.

We do not, in this paper, settle the question of whether or not Hilbert's second axiom is adequate for three-space geometries. This question is bound up with an unsolved problem concerning transformation groups.

Finally we wish to remark that instead of assuming that the space we are dealing with is ordinary three-space, it is only necessary to make certain topological assumptions on the space from which it follows by virtue of the

Presented to the Society, October 28, 1939; received by the editors December 20, 1939. 
same group axioms that the space is actually a number-space. But we reserve discussion of this matter for another occasion. In this connection see the abstract by the authors in the Bulletin of the American Mathematical Society, vol. 45 (1939) (no. 349).

2. The axioms. We formulate two sets of axioms, the first set for the plane, and the second set for three-space. The first set, to which we proceed immediately, is the set used by Hilbert except that it has been materially weakened in the manner described.

We assume then that there is given a system $\left(E_{2}, G\right)$ where $E_{2}$ is the number-plane and $G$ is a set of sense preserving homeomorphisms of this plane, and that this set satisfies the following conditions:

\subsection{The system $G$ is a group.}

The assumption tacitly implicit in the above is that $G$ is effective, that is, that no element except the identity leaves all of $E_{2}$ fixed.

With each point $x$ in space there exists a subgroup $G_{x}$ consisting of elements of $G$ which leave $x$ fixed.

2.2. If $x$ is any point of $E_{2}$ and $y$ is distinct from $x$, then $G_{x}(y)$ is infinite.

This axiom could be reformulated so that it would be entirely analogous to our axiom $2.2^{\prime}$ for three-space but we do not carry this out. It would involve almost no change in the work.

2.3. Let $(x, y)$ and $\left(x^{\prime}, y^{\prime}\right)$ be two pairs of points of $E_{2}$, where the points of a pair are not necessarily distinct. If there exist pairs $\left(x_{n}, y_{n}\right)$ and $\left(x_{n}^{\prime}, y_{n}^{\prime}\right)$, the first arbitrarily near $(x, y)$, the second arbitrarily near to $\left(x^{\prime}, y^{\prime}\right)$, and if there exists an element of $G$ taking $\left(x_{n}, y_{n}\right)$ to $\left(x_{n}^{\prime}, y_{n}^{\prime}\right)$, then there exists an element of $G$ taking $(x, y)$ to $\left(x^{\prime}, y^{\prime}\right)$.

As we have said, this set of axioms is exactly Hilbert's except that the third axiom has been weakened to a condition on pairs instead of triples of points.

We now formulate our axioms for three-space. We assume that there is given a system $\left(E_{3}, G\right)$ where $E_{3}$ is ordinary three-space and $G$ is a set of sense preserving homeomorphisms of $E_{3}$ satisfying the following conditions:

2.1'. The same as 2.1 .

$2.2^{\prime}$. There exists a point $p$ of $E$ such that the group $G_{p}$ is a proper subgroup of $G$ and for a sequence of points $p_{n}$ approaching $p$ the sets $G_{p}\left(p_{n}\right)$ are at least two dimensional.

\section{$2.3^{\prime}$. The same as 2.3 .}

Occasionally we shall refer to the situation described by the first set of axioms as the plane case and to the situation described by the second set as 
the space case. In both cases we shall prove that ordinary geometric concepts such as "line" and "distance" (and in the space case "plane") may be defined in terms of $G$ in such a way that we obtain either euclidean or hyperbolic geometry and that $G$ is the group of rigid motions of the corresponding geometry we obtain. We do this in the plane case by proving Hilbert's axioms [5], that is, by proving that the two-point condition 2.2 implies the three-point condition. The space case we treat in detail and show in detail that there are only the two systems.

Our approach to this problem differs from Hilbert's in one important respect. Hilbert analyzes more or less directly the topological nature of the orbit $G_{p}(x)$. In three-space this course seems to us not feasible until much more is known about strongly homogeneous subsets of space. But even granting such knowledge our procedure has the advantage of making available the results of topological group theory. Thus, we proceed at once to a study of the group $G_{p}$ as a topological transformation group. In brief summary, we first confine our attention to a suitable invariant neighborhood of the point $p$ where orbits under $G_{p}$ can be proved compact. We form the effective group in this neighborhood, and show that any sequence of elements of this group has a subsequence which converges to a homeomorphism of the neighborhood in to itself. We then augment our group by the addition of such homeomorphisms. It transpires, only considerably later, that this enlargement is an illusory one. The enlarged group is then shown to be a compact topological transformation group on a "locally euclidean" space. From our previous work, we then know that our orbits are necessarily manifolds, and it is not difficult to show that they are indeed spheres. From an earlier paper of ours we learn also the complete structure of the group and its behavior in the neighborhood. We are now in a position to show, by an argument patterned on one of Hilbert's, that the neighborhood above coincides with space.

The use of a "two-point" rather than "three-point" axiom shows itself in one or two interesting ways in the study of $G_{p}$ but becomes a matter of considerable moment in the study of the group $G$ as a topological transformation group of the space. This argument is given in $\$ 12$. To this point the case of the plane or of space may be treated more or less simultaneously, and it seems to us, in fact, that much of this generalizes with no great difficulty to fourspace and perhaps farther.

The remainder of the paper is devoted to a study of the geometry induced in space by the group $G$. Here, after a few paragraphs, we are on ground already explored by Kerékjártó. His paper was not known to us until our own had been completed, and we carry out the program essentially as we had it. We do this in part for completeness sake, in part because the form in which our solution is set differs sufficiently from Kerékjártó's. At one point in proving the linearity of our planes we borrow a very ingenious idea from Kerékjártó's paper which shortens considerably an argument of our own. 
3. In a great part of the paper we treat the two cases simultaneously, calling the space (which of course is either $E_{2}$ or $E_{3}$ ) simply $E$. When we speak of a sphere or a rotation group we of course mean the one appropriate to the dimension of the space.

Let $p$ be a point of $E$, which for $E_{2}$ may be any point, but which for $E_{3}$ is to be the point specified by $2.2^{\prime}$.

Hilbert points out that for any $x$ the set $G_{p}(x)$ is closed. This is a consequence of 2.3. Thus: let $x_{n}$ be a sequence of points in $G_{p}(x)$ converging to a limit point $x_{0}$. There are elements $g_{n}$ in $G_{p}$ such that $x_{n}=g_{n}(x)$. The element $g_{n}$ takes the pair $(p, x)$ to the pair $\left(p, x_{n}\right)$. By 2.3 there is a $g$ in $G$ which takes $(p, x)$ to $\left(p, x_{0}\right)$ and this element certainly is in $G_{p}$.

By a similar method it is seen that $G(x)$, which ultimately will be shown to coincide with $E$, is closed.

4. Let $R$ be the set of points $x$ such that $G_{p}(x)$ is compact. This set is not vacuous for it contains $p$.

\section{Lemma 1. The set $R$ is open.}

Let $x$ be any point in $R$, and let $B$ be a conditionally compact open set containing $G_{p}(x)$ in its interior. Let $S$ be the boundary of $B$. We assert that there is an open set $V$ containing $G_{p}(x)$ such that $G_{p}$ carries no point of $S$ inside $V$. Otherwise there must exist a set of elements $g_{n}$ in $G_{p}$ and a set of points $b_{n}$ in $S$ such that at least one point of the set $g_{n}\left(b_{n}\right)$ lies in every neighborhood of the compact set $G_{p}(x)$. There is no loss in assuming that $b_{n}$ approaches some point $b$ in $S$ and $g_{n}\left(b_{n}\right)$ approaches a point $a$ in $G_{p}(x)$. But then there must be an element of $G_{p}$ taking $b$ to $a$ which means that a point of $S$ is in $G_{p}(x)$ contrary to the choice of $B$.

Let $W$ denote those components of the above determined $V$ which include points of $G_{p}(x)$. The set $W$ is open and no element of $G_{p}$ carries a point of $W$ outside of $B$. For such an element would also leave an element of $W$ inside $B$ (any point namely in which $W$ meets $G_{p}(x)$ ), and it would therefore carry some point of $W$ into $S$ which is impossible.

It has now been shown that all points of $W$ have orbits inside $B$. Therefore every point of $W$ has a compact orbit and $x$ is in an open set $W$ all of whose points have compact orbits as the lemma demands.

4.1. The proof shows even more than is required in Lemma 1. It shows, for any point $x$ in $R$, that $x$ is an interior point of a set $W$, such that $G_{p}(W)$ is a conditionally compact set. If $\bar{W}$ is the closure of $W$, then $G_{p}(\bar{W})$ is compact and $x$ is seen to be an interior point of a set $\bar{W}$ such that $G_{p}(\bar{W})$ is compact. These facts together with the Heine-Borel theorem enable us to state the following lemma.

LEMMA 2. If $M$ is any compact subset of $R$, then $G_{p}(M)$ is compact.

5. We consider now the action of $G_{p}$ on $R$. Conceivably $G_{p}$ has a non- 
trivial subgroup leaving all of $R$ fixed. Later this possibility will be ruled out, but meanwhile we must take account of it. Let $G_{p}^{1}$ be the subgroup leaving all of $\bar{R}$ fixed. This subgroup includes the identity at any rate, and $G_{p} / G_{p}^{1}$, which will be denoted for brevity by $H$, is an effective transformation group of $\bar{R}$.

Our next task is to show that $H$ may be extended to become a compact transformation group of $\bar{R}$. It will be assumed that $E$ is assigned a bounded metric, say the metric of a three-sphere or a two-sphere according to the case, which is obtained by adding a point to $E$. This means that we can define a distance between any two transformations of $E$ into itself or between any two transformations of a subset of $E$ into itself. For example if $f$ and $g$ are two transformations of $\bar{R}$ into itself

$$
d(f, g)=\text { l.u.b. } d[f(x), g(x)]
$$

where $x$ ranges over $\bar{R}$. Under this distance $H$ becomes a metric space.

5.1. Lemma 3. If a sequence of elements of $G$ converges everywhere on a set $M$ to a limit $h$, then $h$ is continuous on $M$. If $M$ is compact the convergence is uniform.

Let us prove first that $h$ is continuous. Let $m$ be any point of $M$ and let $S$ be any sphere with $f(m)$ as a center. Let $m_{i}$ be a sequence of points of $M$ approaching $m$. We shall show that almost all the points $h\left(m_{i}\right)$ are inside or on $S$. Assume that this is not true for an infinite subsequence, say $m_{k_{i}}$, and let $m_{k_{i}} m$ be a short arc joining $m_{k_{i}}$ to $m$. Since $g_{n}$ approaches $h$ and since $h\left(m_{k_{i}}\right)$ is outside $S$ by assumption, there will certainly be an integer, say $n_{i}$, such that $g_{n_{i}}\left(m_{k_{i}}\right)$ is outside $S$. We may assume without loss of generality that every $g_{n_{i}}(m)$ is inside $S$. Hence there is a point, say $y_{k_{i}}$, on the arc $m_{k_{i}} m$ such that $g_{n_{i}}\left(y_{k_{i}}\right)$ is on $S$. Assume that $g_{n_{i}}\left(y_{k_{i}}\right)$ approaches a point $b$ on $S$. Then $g_{n_{i}}$ takes the pair $\left(y_{k_{i}}, m\right)$ which is near $(m, m)$ to the pair $\left[g_{n_{i}}\left(y_{k_{i}}\right), g_{n_{i}}(m)\right]$ which is near $[b, h(m)]$. This is a contradiction from which the continuity of $h$ follows.

We shall next show that the convergence is uniform in case $M$ is compact. If the convergence is not uniform, there is for some positive number $e$ an infinite set of indices $k_{1}, k_{2}, \cdots$ and a set of points $m_{1}, m_{2}, \cdots$ in the set $M$ such that

$$
d\left[g_{k_{i}}\left(m_{i}\right), h\left(m_{i}\right)\right] \geqq e .
$$

There is no loss in assuming that the sequence $m_{i}$ converges to $m$ and that $g_{k_{i}}\left(m_{i}\right)$ converges to a point $b$. From the above inequality $b$ and $h(m)$, which is the limit of $h\left(m_{i}\right)$ by the continuity, are distinct. The transformations $g_{k_{i}}$ therefore take the pair $\left(m_{i}, m\right)$ which is near $(m, m)$ to the pair $\left[g_{k_{i}}\left(m_{i}\right)\right.$, $\left.g_{k_{i}}(m)\right]$ which is near $[b, h(m)]$. By 2.3 there must be an element of $G$ taking $m$ to each of the distinct points $b$ and $h(m)$. This contradiction shows that the convergence is uniform. 
LEMMA 3.1. Let $g_{n}$ be a sequence of elements of $G$ converging to $h$ everywhere on a set $M$. Then if $m_{i}$ approaches $m$ it follows that $g_{i}\left(m_{i}\right)$ approaches $h(m)$.

The set $B$ containing the points $m$ and all $m_{i}$ 's is compact. Hence on this set $g_{n}$ converges uniformly to $h$. Let $e$ be any positive number. For all $n$ greater than an integer $N_{1}$,

$$
d\left[g_{n}(x), h(x)\right]<e / 2
$$

for all $x$ in $B$. Since $h$ is continuous, there will be an integer $N_{2}$ such that if $n$ is greater than $N_{2}$

$$
d\left[h\left(m_{n}\right), h(m)\right]<e / 2 .
$$

For all $n$ greater than $N_{1}$ and $N_{2}$ we have not only this latter inequality but we have as a consequence of the first inequality

$$
d\left[g_{n}\left(m_{n}\right), h\left(m_{n}\right)\right]<e / 2 .
$$

The last two inequalities yield the desired conclusion.

LEMMa 3.2. If a sequence $f_{n}$ of elements of $G_{p}$ converges everywhere on a compact set $M$, invariant under $G_{p}$, to a transformation $f$, then $f$ is a homeomorphism of $M$ into itself.

In view of Lemma 3 it is only necessary to show that $f$ has a single valued inverse and that $f(M)=M$.

If $f$ does not have a single valued inverse, there must be two distinct points $b$ and $c$ such that $f(b)=f(c)$. Then the elements $f_{n}$ take the pair $(b, c)$ to the pair $\left[f_{n}(b), f_{n}(c)\right]$ and by 2.3 there is an element in $G$ which takes both $b$ and $c$ to $f(b)$. This contradiction shows that $f$ is one-one, and since $M$ is compact $f$ must take $M$ homeomorphically to $f(M)$. We know that $f(M)$ is a subset of $M$, and to complete the proof of the lemma it remains only to show that $f(M)$ coincides with $M$.

Let $b$ be any point in $M$. Then there is an element $m_{n}$ in $M$ such that $f_{n}\left(m_{n}\right)=b$. Assume that the sequence $m_{n}$ approaches a point $m$. Now $f_{n}$ takes the pair $\left(m_{n}, m\right)$ which is near $(m, m)$ to the pair $\left[b, f_{n}(m)\right]$ which is near $[b, f(m)]$. Hence some element of $G$ takes $m$ to both points $b$ and $f(m)$ which is possible only if $f(m)=b$.

\subsection{Lemma 4. The group-space $H$ defined in $\$ 5$ is conditionally compact.}

Yet $Y$ be a countable dense subset of $R$. Let $g_{n}$ be an infinite sequence of elements of $H$. Strictly speaking the $g_{n}$ 's are not elements of $G_{p}$ but there are elements of $G_{p}$ coinciding with these elements on $\bar{R}$, and properties of the group $G_{p}$ may be used in examining the $g_{n}$ 's.

For any point $y$ in $Y$ the sequence $g_{n}(y)$ is conditionally compact and has a convergent subsequence. The limit of this sequence belongs to $G_{p}(y)$ and is a 
point of $R$. By the diagonal process there exists a subsequence $f_{n}$ of the elements $g_{n}$ such that $f_{n}(y)$ converges to a unique point of $R$ for every element $y$ of $Y$. Then, on $Y$, the sequence $f_{n}$ converges to a pointwise limit function $f$.

It will now be shown that $f$ is uniformly continuous in every conditionally compact open subset $R_{1}$ of $R$. In order to do this it must be shown that for every positive $e$ there exists a positive $d$ such that whenever $y$ and $y^{\prime}$ of $Y \cdot R_{1}$ are nearer to each other than $d$, the corresponding $f(y)$ and $f\left(y^{\prime}\right)$ are nearer than $e$. If this is not the case, there must exist in $Y$ a sequence of points $y_{n}$ and $y_{n}^{\prime}$ which may be supposed to converge to the same point $z$ of $\bar{R}_{1}$ such that $f\left(y_{n}\right)$ and $f\left(y_{n}^{\prime}\right)$ also converge and converge to two distinct points $y$ and $y^{\prime}$ in $\bar{R}_{1}$. This means that some of the elements $g_{n}$ take a pair of points near $z$ to a pair near $\left(y, y^{\prime}\right)$. By 2.3 there is an element of $G$ carrying the point $z$ to the two points $y$ and $y^{\prime}$. This is manifestly impossible and the contradiction establishes the uniform continuity of $f$ on the set $R_{1} \cdot Y$.

This uniform continuity of $f$ permits us to extend it, and we assume it is so extended, to a single valued continuous transformation of $R$ (which of course is locally compact) into itself. The sequence $f_{n}$, which originally was known to converge only on $Y$, is now seen to converge everywhere on $R$ to the transformation $f$. By Lemma 3 the convergence is uniform on compact sets which implies that the sequence $f_{n}$ of elements of $H$ must be a Cauchy sequence because our metric brings two functions close which agree closely outside of a neighborhood of "infinity."

5.3. When we speak of a topological transformation group we use the term with the definition as given in our papers referred to in the bibliography.

Lemma 5. The group $H$ may be extended to a compact group $H$ which is an effective topological transformation group of $R$.

The space $H$ is conditionally compact so that if the space is made complete the resulting space $\bar{H}$ will be compact. The preceding lemmas and their proofs show that Cauchy sequences of $H$ will actually converge to homeomorphisms of $R$ into itself. The space $\bar{H}$ is therefore a transformation group of $R$. To be sure that it is a topological transformation group we must prove that if $g_{n}$ approaches $g$, these being elements in $\bar{H}$, and $x_{n}$ approaches $x$ in $R$ then $g_{n}\left(x_{n}\right)$ approaches $g(x)$. This follows as in the proof of Lemma 3.1.

That $\bar{H}$ is an effective group follows from the fact that distinct elements of the space $\bar{H}$ arise from nonequivalent Cauchy sequences and these give rise to distinct limiting transformations.

6. The present section contains some simple, purely group theoretical, considerations which will be of use to us later.

THEOREM 1. The only two dimensional manifolds which are coset spaces (orbits) of a compact connected group $H$ are the two-sphere, torus, and projective plane. 
There is no loss in assuming that $H$ is effective in its action on the manifold so that $H$ is a Lie group [10]. The theorem then follows from the corresponding theorem on Lie groups due to Cartan [2].

We note without giving the proof, which is not difficult, the following: If a circle group operates on a torus and has a fixed point, then it must leave every point of the torus fixed.

THEOREM 2. The only compact group of sense preserving transformations which can act effectively and transitively on a two-sphere $M$ is the group of rigid rotations of the two-sphere.

For connected groups the theorem is true [9]. If $H$ is the group, let $H^{*}$ be the identity component of $H$, and let $x$ be a point of the sphere. The dimension of $H^{*}(x)$ is the same as the dimension of $H(x)$, namely two, and therefore $H^{*}(x)$ coincides with $M$. Since $H^{*}$ is connected it must be the twosphere rotation group. Assuming $H^{*}$ is not all of $H$ means that, for some $y$, $\left(H^{*}\right)_{y}$ is a proper subgroup of $H_{y}$ because every element of $H$, being sense preserving, has a fixed point; the connectedness of the group $\left(H^{*}\right)_{y}$ (it is of course circular) shows that it is the component of the identity of $H_{y}$. Then $\left(H^{*}\right)_{y}$ is invariant in $H_{y}$. Let $M^{*}$ be the decomposition space of $M$ under $\left(H^{*}\right)_{y}$. The group $H_{y} /\left(H^{*}\right)_{y}$ acts on this space which is an interval. Hence $H_{y} /\left(H^{*}\right)_{y}$ contains only the identity, or it is a group of two elements which merely interchanges the ends of $M^{*}$ while leaving a "middle" point fixed. The latter possibility cannot occur, for if it did $H_{y}$ would contain an element moving $y$. Hence $H_{y} /\left(H^{*}\right)_{y}$ contains only the identity element and $\left(H^{*}\right)_{y}$ is not a proper subgroup of $H_{y}$ as we assumed. The contradiction shows that $H^{*}$ coincides with $H$ and that $H$ is the group of rigid rotations of the two-sphere.

COROLLARY. The only compact group of sense preserving transformations of three-space into itself with at least one two dimensional orbit is the two-sphere rotation group.

7. In the present section we confine our attention to the space case. The groups $H$ and $G_{p}$ have the same orbits in $R$, and since these orbits are closed $\bar{H}$ has the same orbits as do $H$ and $G_{p}$. Let $H^{*}$ be the component of the identity of $\bar{H}$. The orbit of a point under $H^{*}$ will have the same dimension as the orbit of the point under $\bar{H}$. Hence $H^{*}$ has a sequence of two dimensional orbits approaching the fixed point $p$. We will now consider the action of the effective compact transformation group $H^{*}$ on the connected locally "euclidean" space $R^{0}$, where $R^{0}$ denotes that component of $R$ which contains $p$. (It is conceivable that some subgroup of $H$ should leave all of $R^{0}$ fixed. We assume without changing our notation that this is not the case. There is no loss in this process as we might as well have assumed we were working with $R^{0}$ before.)

It follows from theorems on the structure of coset spaces [10] that any two dimensional orbit of $H^{*}$ in $R^{0}$ must be a two dimensional manifold. Any 
two dimensional orbit of $H^{*}$ in $R^{0}$ must be, therefore, one of three types of manifold, the two-sphere, the projective plane, or the torus. The projective plane cannot be imbedded in $E$, so that the number of possibilities is reduced immediately to two, the sphere and torus.

In the orbit space associated with $R^{0}$, call it $R^{*}$, every two dimensional orbit is a cut point of order two precisely. The set of such orbits is open [10]. The space $R^{*}$ has one non-cut point, at least, namely the orbit consisting of the point $p$ only. By the cyclic element theory, $R^{*}$ must be either a line, a ray, or an interval. It cannot be a line because it contains a non-cut point. It cannot be an interval for this would mean that $R^{0}$, an open subset of $E$, would be compact. Hence $R^{*}$ is a ray, and this shows that all orbits in $R^{0}$, with the exception of $p$, are two dimensional orbits which are either spheres or tori. The group $\bar{H}$ can be seen to be a Lie group because it operates on a locally euclidean connected space with locally connected orbits [10]. It will now be of dimension three at most [10], and it will be effective on each one of its two dimensional orbits. For, if a subgroup left all of a manifold orbit fixed this same subgroup would leave the whole space fixed by a simple application, as in an earlier paper of ours [8], of a theorem of Newman. If all two dimensional orbits are spheres, then $H^{*}$ is the rotation group of a sphere, for this is the only connected compact effective transformation group of a sphere. If all two dimensional orbits are tori, then $H^{*}$ is a two dimensional toral group, for this is the only Lie group which can be effective on a torus.

It is intuitively clear that all orbits must be spheres and we now give the proof. We will show that if one orbit is toral then all orbits are. Assume that one orbit $H^{*}(x)$ is toral. If $H^{*}$ is two dimensional, then $H^{*}$ is a toral group and all orbits are tori. If $H^{*}$ is three dimensional there must be a circular subgroup $K$ leaving $x$ fixed. But if a circular subgroup leaves one point of a torus fixed it must leave every point fixed. Hence $K$ leaves all of $H^{*}(x)$ fixed and, since this separates space, we see by a familiar device that $K$ leaves all of space fixed. In this case $H^{*}$ is not effective. We are therefore led to conclude that all two dimensional orbits are tori.

This last situation is impossible. For if $H^{*}$ is a toral group we can form a true section $B$ of the space, that is, we can find a closed set $B$ which has one and only one point on each orbit in $R$. The set $B$ will have to be homeomorphic to $R^{*}$ and will be a ray. Now let $H^{*}(x)$ be a toral orbit inside a neighborhood $U$ of $p$ which is homeomorphic to three-space. There will be in $U-H^{*}(x)$ a one-cycle $Z$ which does not bound in $U-H^{*}(x)$ and which is outside $H^{*}(x)$; that is, it is contained in the component of $U-H^{*}(x)$ which does not contain $p$. Then using the true section $B$ we may deform $H^{*}(x)$ to the point $p$. The cycle $Z$ certainly bounds in $U-p$, contradicting its choice.

Therefore not every orbit is a toral orbit and $H^{*}$ must be the rotation group of three-space, and every two dimensional orbit must be a two-sphere. Let $K$ be a circular subgroup of $H^{*}$. There will have to be precisely two points 
on each two-sphere orbit left fixed by $K$. These two points will define for us a double valued function everywhere on $R^{*}$. The end point of the ray $R^{*}$ is an exception when the function is single valued. But at any rate it is possible to pick out of these functional values a true section $B$ of the entire space. The existence of the ray $B$ proves $R^{0}$ homeomorphic to euclidean three space.

From Theorem 2 of $\S 6$ we see that $\bar{H}$ and $H^{*}$ must coincide, but we can conclude even more.

THEOREM 3. The group $H$ coincides with $\bar{H}$ and is therefore the rotation group of three-space.

Let $x$ be any point of $R$ distinct from $p$. The set $\bar{H}(x)$ is a two-sphere, and $H(x)$ coincides with $\bar{H}(x)$; or in other words $H$, a subgroup of $\bar{H}$, is transitive on the two-sphere. This is possible only if $H$ is all of $\bar{H}$ [11].

8. In this section we turn to the plane case, falling back on Lemma 5 where we left it.

THEOREM 3.1. The group $H$ coincides with $\bar{H}$ and is a circle group, the rotation group of the plane. The set $R^{0}$ is homeomorphic to a plane.

The group $\bar{H}$ cannot be totally disconnected, for such a group cannot operate effectively on a locally planar space (as we have shown [8]). Let $H^{*}$ be the identity-component of $\bar{H}$. As in the space case, the orbits under $H^{*}$ must be manifolds and therefore simple closed curves: they are obviously one dimensional. It follows that $H^{*}$ is a Lie group and in particular the circle group [10]. As in the space case the group $\bar{H}$ must operate upon the decomposition space of $R^{0}$ by orbits under $H^{*}$ : this space is a ray, with $p$ as end point, and $H^{*}$ must be the identity upon it.

Now let $g$ be some element of $\bar{H}$, not the identity. There must be a point $x$ of $R^{0}$ such that $g x$ is not $x$. On the other hand, $g x$ is a point of $H^{*}(x)$ so that for some $g^{\prime}$ of $H^{*}$ we have

$$
g^{\prime} g^{-1} x=x
$$

Since this element is sense preserving and leaves one point of the circle $H^{*}(x)$ fixed, it leaves all $H^{*}(x)$ fixed and then all of $R^{0}$. Therefore it must be the identity and we conclude that $g$ belongs to $H^{*}$. This shows that $\bar{H}$ coincides with $H^{*}$ and is a circle group. Since $H$ is transitive on $\bar{H}(x)$, it is obvious that $H$ must coincide with $\bar{H}$ which is effective on $\bar{H}(x)$.

Now $R^{0}$ is a connected open subset of the plane filled out by a continuous family of simple closed curves and it is intuitively obvious and sufficiently well known that $R^{0}$ must be homeomorphic to the plane.

We turn now to a simultaneous consideration of the two cases. What has been done above is summed up in the following theorem.

THEOREM 4. The group $H$ may be so topologized that it becomes the ordinary 
rotation group of space, and it acts on $R^{0}$, which is homeomorphic to space, as the ordinary rotation group does-in a properly chosen coordinate system.

9. TheOREM 5. The set $R^{0}$ is closed and so coincides with $E$.

The assumption that $R^{0}$ is not closed implies that there is an arc $p x$ which is contained in $R^{0}$ except for its end point which is not in $R^{0}$. Since $x$ is not in $R^{0}$ (and not in $R$ ) there is a sequence of elements $g_{n}$ of $G_{p}$ such that $g_{n}(x)$ tends toward infinity. Let $G_{p}^{R^{0}}$ denote all elements of $G_{p}$ leaving all of $R^{0}$ fixed. Under the homeomorphism taking $G_{p}$ to $G_{p} / G_{p}^{R^{0}}=H$ suppose that $g_{n}$ goes to $\bar{g}_{n}$; assume that the sequence $\bar{g}_{n}$ converges to $\bar{g}$ and that $\bar{g}$ is the image of an element $g$ under this homeomorphism.

Let $S_{1}$ and $S_{2}$ be two spheres each containing $g(p x)$ and such that $S_{2}$ lies inside $S_{1}$. We may assume that all points $g_{n}(x)$ are outside $S_{1}$. On the arc $g_{n}(p x)$ there are two points $g_{n}\left(x_{n}\right)$ and $g_{n}\left(y_{n}\right)$, where $x_{n}$ and $y_{n}$ are points of $p x$, such that $g_{n}\left(x_{n}\right)$ is the first point of $g_{n}(p x)$ on $S_{2}$ and $g_{n}\left(y_{n}\right)$ is the first point of $g_{n}(p x)$ on $S_{1}$. The points $x_{n}$ and $y_{n}$ lie on $p x$ in the order $p x_{n} y_{n} x$.

There is no loss in assuming that the sequences $x_{n}, y_{n}, g_{n}\left(x_{n}\right)$, and $g_{n}\left(y_{n}\right)$ converge respectively to $x^{\prime}, y^{\prime}, x^{*}$ and $y^{*}$.

We wish to prove that $x^{\prime}$ is identical with $x$. If it is not, $x^{\prime}$ must be a point of $R^{0}$ and the points $x_{n}$ may also be taken to be in $R^{0}$. Hence $g_{n}\left(x_{n}\right)$ $=\bar{g}_{n}\left(x_{n}\right)$ converges to $\bar{g}\left(x^{\prime}\right)=g\left(x^{\prime}\right)$ which is impossible because $g\left(x^{\prime}\right)$ is inside $S_{2}$. Hence $x^{\prime}$ and $x$ are identical.

This shows that the points $x_{n}$ converge to $x$ and of course the points $y_{n}$ must also converge to $x$. There are, consequently, elements of $G$ which take the pair $\left(x_{n}, y_{n}\right)$ which is near $(x, x)$ to the pair $\left[g_{n}\left(x_{n}\right), g_{n}\left(y_{n}\right)\right]$ near to the district pair $\left(x^{*}, y^{*}\right)$. There is then an element of $G$ taking $x$ to the two distinct points $x^{*}$ and $y^{*}$. From this contradiction the theorem follows.

COROLlaRy. The group $G_{p}^{R^{0}}=H$, idle on all of $R^{0}$, contains only the identity and the group $G_{p}$ is itself the rotation group of space.

10. Theorem 6. The group $G$ is transitive on the space $E$.

It has already been remarked that $G(x)$ is closed for any $x$ in $E$. In particu$\operatorname{lar} G(p)$ is closed, and in order to prove our theorem it suffices to prove that $G(p)$ is open.

We know in the plane case as well as in the space case that $G_{p}$ is a proper subgroup of $G$ and there must be an element $g$ in $G$ and a point $q$ distinct from $p$ such that $g(p)=q$. The set $G_{p}(q)$ is a sphere. Let $q^{\prime}$ denote another point of $G_{p}(q)$ and let $t$ be a varying element of $G_{p}$ which takes $q$ continuously to $q^{\prime}$. There is a neighborhood $U$ of $q$ so small that its translation $U^{\prime}$ to a neighborhood of $q^{\prime}$ has no point in common with its original position. The set $U^{\prime}$ is the image of $U$ under the terminal element of the parameter $t$.

There is a neighborhood $V$ of $p$ so small that $g(V)$ is inside $U$. As $q$ is 
swept continuously to $q^{\prime}$ it must come in contact with the $g$-image of every sphere about $p$ and within $V$.

Now let $s$ be any point of $V$ and let $S=G_{p}(s)$ be its sphere orbit under $G_{p}$. There is a $t$ such that $t(q)$ is in $g(S)$, that is,

$$
t\{g(p)\}=t(q)=g g^{\prime}(s)
$$

for some $g^{\prime}$ in $G_{p}$. Then

$$
g^{\prime-1} g^{-1} t g(p)=s .
$$

In other words $p$ may be carried to any element of $V$ by some element in $G$. Therefore $p$ is an interior point of $G(p)$ and every point of $G(p)$ must be an interior point.

11. The fact that $G$ is transitive on $E$ tells us the nature of $G_{x}$ when $x$ is distinct from $p$. Let $x=g(p)$. Then $G_{x}=g G_{p} g^{-1}$ and $G_{x}$ is also the rotation group and in a proper system of coordinates "centered" on the point $x$ it acts as the rotation group ordinarily does.

12. Before such geometric concepts as lines and planes can be studied, we need to analyze the nature of $G$ as a topological transformation group. It is in this that we encounter the principal difficulties implicit in our use of the "two-point" form rather than Hilbert's "three-point" axiom. In much of this work we shall continue to treat the plane and space cases together.

Leмma 6. Let $x_{n}$ be a sequence of points converging to $x$ and let $g_{n}$ in $G$ be such that $g_{n}\left(x_{n}\right)$ approaches $x$. Then for any $z$ in $E$, the set $g_{n}(z)$ is bounded.

Let $O$ be the interior of an orbit $S$ of $G_{x}$ which is so large that it surrounds $z$, all of the $x_{n}$ 's, and all of the points $g_{n}\left(x_{n}\right)$. Let $O^{*}$ be the interior of a larger orbit $S^{*}$ so that $S$ is interior to $O^{*}$. Let $z x_{n}$ be arcs of $O$, and suppose now that for infinitely many of the elements $g_{n}$ it is true that $g_{n}(z)$ is outside or on $S^{*}$. For these $n$ 's, and we take it now that all $n$ 's are such, the $\operatorname{arcs} g_{n}\left(x_{n} z\right)$ have one point in $O^{*}$ and one point not in $O^{*}$. They therefore have a first point $z_{n}^{\prime}$ on $S^{*}$. There is a point $x_{n}^{\prime}$ on $x_{n} z$ such that $z_{n}^{\prime}=g_{n}\left(x_{n}^{\prime}\right)$. We may suppose that $x_{n}^{\prime}$ converges to a point $x^{\prime}$ which is in $O$ or $S$, while $z_{n}^{\prime}$ converges to a point $z^{\prime}$ on $S^{*}$. There must be an element $g$ in $G$ which leaves $x$ fixed and carries $x^{\prime}$ to $z^{\prime}$. This is by 2.3 because $g_{n}$ takes the pair $\left(x_{n}, x_{n}^{\prime}\right)$ to the pair $\left[g_{n}\left(x_{n}\right), z_{n}^{\prime}\right]$. However such an element $g$ is in $G_{x}$ and hence leaves $S$ and its interior invariant so that a contradiction has been reached. Hence $g_{n}(z)$ is compact because almost all its elements are inside $S^{*}$.

The orbit $S^{*}$ was subject only to the requirement that it surround $S$ so that the following corollary is true.

CoRollary. Let $x_{n}$ be a sequence of points converging to $x$ and let $g_{n}$ be such that $g_{n}\left(x_{n}\right)=x_{n}$. Let $z$ be any point of $E$. Then any limit point of the set $g_{n}(z)$ is inside or on any orbit of $G_{x}$ which includes $z$ and every $x_{n}$. 
Our task now is the proof of the following theorem.

THeOREM 6.1. Let $x_{n}$ approach $x$ and let $g_{n}^{\prime}$ be elements of $G$ such that $g_{n}^{\prime}\left(x_{n}\right)$ approaches $y$. Then there is a subsequence $g_{n}^{\prime \prime}$ of the $g_{n}^{\prime}$ and an element $g^{*}$ of $G$ such that $g_{n}^{\prime \prime}$ approaches $g^{*}$ (in the sense of pointwise convergence).

The proof of this theorem is rather long and is based on a number of preliminary lemmas to which we now turn. Lemma 3.1 will also be useful here. As usual we use the letter $g$ for an element of $G$ and we use the letter $h$ for a homeomorphism of $E$ which is not known to be an element of $G$. Convergence of homeomorphisms, as the statement of the theorem implies, is always taken in this section in the sense of pointwise convergence, that is, $h_{n}$ converges to $h$ provided that, for each $x, h_{n}(x)$ converges to $h(x)$.

Lemma 6.12. Let $g_{n}$ be a sequence of elements of $G$ converging to a homeomorphism $h$ of $E$. Let $F$ be an arbitrary compact subset of $E$ and let a positive number $e$ be given. Then there exists an integer $N$ such that if $y$ and $z$ are any two points of $F$ for which $d(y, z)<1 / N$, and $n>N$ then

$$
d\left[g_{n}(y), h(z)\right]<e .
$$

The proof of this lemma which is quite similar to various preceding proofs will be omitted.

In the hypotheses of the following lemmas it will frequently occur as it did in the preceding lemma that there is a sequence $g_{n}$ of elements of $G$ converging to a homeomorphism $h$ of $E$. From now on we shall express this fact in abbreviated form by writing $g_{n} \rightarrow h$.

Lemma 6.13. Let $g_{n} \rightarrow h$ and let $i$ be a positive integer. Then it is true that $g_{n}^{i} \rightarrow h^{i}$.

The proof will be made by induction. It is true when $i=1$ and we now assume that it is true for $i-1$.

Let $x$ be any point of $E$ and let $F$ be the set made up of the points $x$, $h^{i-1}(x)$, and $g_{n}^{i-1}(x),(n=1,2, \cdots)$. By the hypothesis of the induction $F$ is compact. Let $e$ be any positive number. By the preceding lemma there is an integer $N$ such that if $y$ and $z$ are in $F$ and $d(y, z)<1 / N$ then for $n>N$

$$
d\left[g_{n}(y), h(z)\right]<e .
$$

On the other hand for sufficiently large $n$, say $n$ greater than $N^{\prime}$,

$$
d\left[g_{n}^{i-1}(x), h^{i-1}(x)\right]<1 / N .
$$

Hence if $n$ is larger than $\left(N, N^{\prime}\right)$ we obtain (letting $y$ be $g_{n}^{t-1}(x)$ and $z$ be $\left.h^{i-1}(x)\right)$

$$
d\left[g_{n}^{i}(x), h^{i}(x)\right]<e
$$


Lemma 6.14. Let $g_{n} \rightarrow h$ and let $g$ be an arbitrary element of $G$. Then $g g_{n} \rightarrow g h$.

For an arbitrary $x$ we know that $g_{n}(x) \rightarrow h(x)$. It is then an immediate consequence of the definition of homeomorphism that $g g_{n}(x) \rightarrow g h(x)$.

LEMMA 6.141. Let $g_{n} \rightarrow h$ and assume that there are elements $g$ and $g^{\prime}$ of $G$ such that $g g_{n} \rightarrow g^{\prime}$. Then $g^{\prime}=g h$ and $h$ is in $G$.

By the preceding lemma $g g_{n} \rightarrow g h$ and hence $g^{\prime}=g h$.

Lemma 6.15. Let $K$ be a simple closed curve and let $T$ be a nonidentical sense preserving homeomorphism with a fixed point. Then there exists a pair of points $x$ and $y$ of $K$ such that $T^{i}(x)=x$ and $T^{i}(y) \rightarrow x$ as $i \rightarrow \infty$.

Choose any moving point $y$. Then $T^{i}(y)$ must approach monotonically a point $x$ which is fixed.

Lемма 6.151. Let $g_{n} \rightarrow h$ and suppose that, for a definite point $q$ of $E$, $h(q)=q$. Then $h$ must leave invariant every orbit of $G_{q}$, that is, for all $x$,

$$
h G_{q}(x)=G_{q}(x)
$$

Let $x$ be any point. Since $g_{n} \rightarrow h$ we know that $g_{n}(x) \rightarrow h(x)$ and $g_{n}(q) \rightarrow h(q)$ $=q$. Hence there is an element of $G$ taking $q$ to $q$ and $x$ to $h(x)$. This element is in $G_{q}$ and we have thus shown that $h G_{q}(x)$ is in $G_{q}(x)$. The equality must hold because of the nature of $G_{q}(x)$ in the two cases.

LEMMA 6.152. Let $x_{n}$ be a sequence of points converging to a point $x$ of $E$. Let $g_{n}$ of $G$ be such that $g_{n}\left(x_{n}\right) \rightarrow x$. Then there exists a homeomorphism $h$ of $E$ such that $g_{n}^{\prime} \rightarrow$ for a subsequence $g_{n}^{\prime}$ of $g_{n}$.

The proof here is similar to that of Lemma 4, but it depends also on Lemma 6.

LemMA 6.16. Let $x_{n} \rightarrow x$ and let $g_{n}^{\prime}$ be such that $g_{n}^{\prime}\left(x_{n}\right)=y_{n} \rightarrow y$. Let $g$ (in $G$ ) be such that $g(y)=x$. Then $g_{n}=g g_{n}^{\prime}$ has a subsequence $g_{n}{ }^{\prime \prime}$ which converges to a homeomorphism $h$ and $h(x)=x$.

Since $y_{n} \rightarrow y, g\left(y_{n}\right) \rightarrow g(y)=x$. Then $g_{n}\left(x_{n}\right)=g g_{n}^{\prime}\left(x_{n}\right)=g\left(y_{n}\right) \rightarrow x$. By the preceding lemma there is a subsequence $g_{n}^{\prime \prime}$ and a homeomorphism $h$ such that $g_{n}^{\prime \prime} \rightarrow h$. By Lemma 3.1, $g_{n}^{\prime \prime}\left(x_{n}\right) \rightarrow h(x)$ and hence $h(x)=x$.

We are now ready to prove Theorem 6.1 for the plane and we restate it here for this case.

Theorem 6.1'. Let $x_{n} \rightarrow x$ and let $g_{n}^{\prime}$ be elements of $G$ such that $g_{n}^{\prime}\left(x_{n}\right) \rightarrow y$. Then there is a subsequence $g_{n}^{\prime \prime \prime}$ of the $g_{n}^{\prime}$ and an element $g^{*}$ of $g$ such that $g_{n}^{\prime}{ }^{\prime} \rightarrow g^{*}$. (For $E_{2}$.)

Let $g_{n}^{\prime \prime}$ and $h$ be as in Lemma 6.16. The elements $g_{n}^{\prime \prime}$ are sense preserving 
and consequently $h$ is sense preserving. For all $z$ distinct from $x, G_{x}(z)$ is a simple closed curve. Since $h G_{x}(z)$ is in $G_{x}(z)$ and since $h$ is a homeomorphism, $h G_{x}(z)=G_{x}(z)$ and $h$ is sense preserving on $G_{x}(z)$ (see Lemma 6.151).

For convenience let $K=G_{x}(z)$ for an arbitrary point $z$ distinct from $x$, and let $z^{\prime}=h(z)$. Since $G_{x}$ is transitive on $K$, there is a $g^{\prime}$ in $G_{x}$ such that $g^{\prime}\left(z^{\prime}\right)=z$, that is, $g^{\prime} h(z)=z$. By a previous lemma $g^{\prime} g_{n}^{\prime \prime} \rightarrow g^{\prime} h$. Since $g^{\prime}$ is in $G_{x}$,

$$
g^{\prime} h(x)=g^{\prime}(x)=x \text {. }
$$

It follows that $h^{\prime}=g^{\prime} h$ preserves the orbits of $G_{x}$ and in particular it follows that $h^{\prime}$ is a sense preserving homeomorphism of $K$. Moreover $h^{\prime}(z)=z$.

We wish to prove that $h^{\prime}$ is the identity on $E$ and we begin by proving that $h^{\prime}$ is the identity on $K$.

If $h^{\prime}$ is not the identity, then there is a pair of distinct points $x^{*}$ and $y^{*}$ such that $h^{\prime i}\left(x^{*}\right)=x^{*}$ and $h^{\prime i}\left(y^{*}\right)$ approaches $x^{*}$ as $i$ approaches infinity. For a fixed $i$ we know that $g_{n}^{* i} \rightarrow h^{\prime i}$ where $g_{n}^{*}=g^{\prime} g_{n}^{\prime \prime} \rightarrow g^{\prime} h=h^{\prime}$. In view of these two facts we see that $g_{n}^{* i}$ for some $n$ and $i$ takes $x^{*}$ and $y^{*}$ into a specified neighborhood of $x^{*}$ which violates the two-point condition. Hence $h^{\prime}$ is the identity on $K$.

We shall show next that the set of points of $E-x$ on which $h^{\prime}$ is the identity contains only inner points. Let $z^{\prime}$ be any point in $E-x$ which is fixed under $h^{\prime}$. Since $h^{\prime}\left(z^{\prime}\right)=z^{\prime}, h^{\prime}$ must leave invariant the orbits $G_{z^{\prime}}\left(z^{*}\right)$ for any $z^{*}$ in $E$. Let $K^{\prime}=G_{x}\left(z^{\prime}\right)$. By the argument above we see that $h^{\prime}$ leaves every point of $K^{\prime}$ fixed. Let $U$ be a neighborhood of $z^{\prime}$ so small that points of $K^{\prime}$ lie outside of $U$ and let $V$ be a neighborhood of $z^{\prime}$ which is invariant under $G_{z^{\prime}}$ and is contained in $U$. For any point $z^{*}$ of $V$ the simple closed curve $K^{*}=G_{z^{\prime}}\left(z^{*}\right)$ must contain a point of $K^{\prime}$. This is a fixed point of $h^{\prime}$ and the argument of the preceding paragraph shows that every point of $K^{*}$ is fixed under $h^{\prime}$. Then it follows that all of $V$ is fixed under $h^{\prime}$.

Hence the set of points fixed under $h^{\prime}$ is both open and closed in $E-x$. This set of fixed points therefore includes $E-x$ and since it is closed it must include $E$. Hence $h^{\prime}$ is the identity.

Now $h^{\prime}=g^{\prime} h$ so that $h=g^{\prime-1}$ and $h$ is an element of $G_{x}$. This completes the proof of the theorem for the case of the plane. To see this assume for convenience that $g_{n}^{\prime \prime}$ and $g g_{n}^{\prime}$ coincide. Then $g g_{n}^{\prime} \rightarrow h=g^{\prime-1}$ and $g_{n}^{\prime} \rightarrow g^{-1} g^{\prime-1}$.

We turn next to the proof of the theorem for $E_{3}$.

TheOREM 6.1' ${ }^{\prime \prime}$. Let $x_{n} \rightarrow x$ and let $g_{n}^{\prime}$ be elements of $G$ such that $g_{n}^{\prime}\left(x_{n}\right) \rightarrow y$. Then there is a subsequence $g_{n}^{\prime \prime}$ of $g_{n}^{\prime}$ and an element $g^{*}$ of $G$ such that $g_{n}^{\prime \prime \prime} \rightarrow g^{*}$. (For $E_{3}$.)

Let $g_{n}^{\prime \prime}$ and $h$ be as in the preceding proof, that is, as in Lemma 6.16. The transformations $g_{n}^{\prime \prime}$, and therefore $h$ also, are sense preserving in $E$. The point $x$ is fixed under $h$ and $h$ preserves orbits $G_{x}(z)$, these orbits being twospheres. Let $z$ be a definite point of $E$ distinct from $x$ and let $S=G_{x}(z)$. Since 
$h$, being sense preserving, has at least one fixed point on $S$ we may assume that $z$ is such a fixed point. Then $h$ will preserve orbits $G_{z}(y)$ for all $y$ of $E$.

We are going to look for some simple closed curve $K$, on $S$, invariant under $G_{x}$ and $G_{z}$ and also invariant under $h$. Let $S^{\prime}$ denote a sphere orbit under $G_{z}$ such that some points of $S$ are outside $S^{\prime}$. Let $F$ denote the closed intersection of $S$ and $S^{\prime}$ and let $D$ denote the component of $S-F$ which contains $z$.

Now $S$ and $S^{\prime}$ are invariant under $h$ and their intersection $F$ must also be invariant under $h$. Hence $S-F$ is invariant and $D$ must be invariant under $h$ since it is a component with a fixed point. Let $K$ be the boundary of $D$ on $S$. It is this set which will be shown to be a simple closed curve. Observe at the moment that $K$ is invariant under $h$ and is a subset of $F$.

The group $G_{x z}$ leaving $x$ and $z$ fixed is a circle group and $F$ is invariant under $G_{x z}$. Let $y$ denote any point of $K$ above. Then $K=G_{x z}(y)$. Hence $K$ is a simple closed curve invariant under $h$. Furthermore $h$ in its action on $K$ must be sense preserving for otherwise it would have to interchange the two components of $S-K$ which it cannot do since we know that $D$ contains a fixed point.

Since $G_{x z}$ is transitive on $K$ there is an element $g^{\prime} h=h^{\prime}$ which has all the properties of $h$ which we need to use and in addition has a fixed point on $K$.

We wish to show that $h^{\prime}$ is the identity on $S$ ultimately enlarging the set of fixed points to include all of $E$. By a familiar argument $h^{\prime}$ must leave all of $K$ fixed. The following lemma will be useful to us as we proceed.

Lemma 6.17. If $h^{\prime}$ leaves fixed a point $p$ and a continuum on an orbit $S^{*}=G_{p}(q)$, then $h^{\prime}$ leaves all of $S^{*}$ fixed.

Let $F$ be the set of fixed points of $h^{\prime}$ on $S^{*}$, and let $M=S^{*}-F$. Let $O$ be a component of $M$, and let $B$ be the boundary of $O$. Now from the hypothesis $B$ cannot be zero dimensional, for if it were $B$ would be all of $F$ and so $F$ would not contain a continuum. Hence $B$ is one dimensional, and it must contain a continuum $C$ which contains a point $b$ which is accessible from $O$. Let $U$ be a neighborhood of $b$ (in $S^{*}$ ) so small that any simple closed curve in $U$ surrounding $b$ must meet $C$. Let $G_{b}(y)$ be an orbit so small that its intersection with $S^{*}$ is in $U$. Then by an argument given above there is a simple closed curve in the intersection of $G_{b}(y)$ and $S^{*}$ which is invariant under $h^{\prime}$. Furthermore $h$ preserves sense on this curve. Since this curve surrounds $b$ it must meet $C$ and consequently $h^{\prime}$ must leave all the curve fixed. As this curve was arbitrarily small we see that $b$ cannot be accessible from $O$. This is a contradiction which proves the lemma.

We can of course conclude now that $h^{\prime}$ leaves all of $S$ fixed. Furthermore any small "sphere" with center on $S$ will also be left fixed by $h^{\prime}$ since it will intersect $S$ in a one dimensional set. The set of "spheres" about $x$ forms a ray (the end point of the ray being of course a point orbit). The above considera- 
tions show that the "spheres" of this ray left entirely fixed by $h^{\prime}$ form a set which is both open and closed. Therefore $h^{\prime}$ leaves every point of $E$ fixed and is the identity.

Now as in the plane case $h=g^{\prime} h$ and $h=g^{\prime-1}$ so that $h$ is in $G$ and indeed in $G_{x z}$. This completes the proof as in the former case.

\subsection{Lemma 6.2. If $g_{n} \rightarrow g$, then $g_{n}^{-1} \rightarrow g^{-1}$.}

Let $x$ be any point of $E$. By hypothesis $g_{n}(x) \rightarrow g(x)$. Also $g_{n} g^{-1}(x) \rightarrow g g^{-1}(x)$ $=x$. If we knew that $g g_{n}^{-1}(x) \rightarrow x$ we could conclude that $g_{n}^{-1}(x) \rightarrow g^{-1}(x)$. The proof of the lemma is therefore reduced to the proof of the following special case. If $g_{n}$ is in $G$ and $g_{n}(x) \rightarrow x$, then $g_{n}^{-1}(x) \rightarrow x$.

In order to prove this proposition let $S$ be any sphere about $x$. We shall show that almost all the points $g_{n}^{-1}(x)$ are inside $S$. If this is not true we arrive at a contradiction as follows. For each $g_{n}^{-1}(x)$ not inside $S$ choose a short arc joining $x$ to $g_{n}(x)$, call it $x g_{n}(x)$. Then $g_{n}^{-1}\left(x g_{n}(x)\right)$ will be an arc joining $g_{n}^{-1}(x)$ to $x$. It will therefore contain a point $y_{n}$ on $S$. Now $g_{n}$ takes the pair $\left(y_{n}, x\right)$ to a pair $\left(g_{n}\left(y_{n}\right), g_{n}(x)\right)$ both elements of this pair being near $x$. The points $y_{n}$ will have a limit $y$ on $S$ and there will have to be a $g$ in $G$ taking both $y$ and $x$ to $x$. This contradiction establishes the lemma.

Lemma 6.3. If $g_{n} \rightarrow g$ and $g_{n}{ }^{\prime} \rightarrow g^{\prime}$, then $g_{n} g_{n}{ }^{\prime} \rightarrow g g^{\prime}$.

Let $x$ be any point of $E$. Then $g_{n}^{\prime}(x) \rightarrow g^{\prime}(x)$. Hence $g_{n}\left[g_{n}^{\prime}(x)\right] \rightarrow g g^{\prime}(x)$ which we wanted to prove.

Theorem 6.2. Let $\left(a_{1}, \cdots, a_{k}\right)$ and $\left(A_{1}, \cdots, A_{k}\right)$ be any two sets of $k$ points. Let $\left(a_{1}^{n}, \cdots, a_{k}^{n}\right)$ approach $\left(a_{1}, \cdots, a_{k}\right)$ and let $\left(A_{1}^{n}, \cdots, A_{k}^{n}\right)$ approach $\left(A_{1}, \cdots, A_{k}\right)$. If there is for each $n$ an element $g_{n}$ in $G$ such that

$$
g_{n}\left(a_{i}^{n}\right)=A_{i}^{n}, \quad i=1, \cdots, k,
$$

then there exists an element $g$ in $G$ such that

$$
g\left(a_{i}\right)=A_{i}, \quad i=1, \cdots, k .
$$

Let $g_{n}^{\prime}$ be an element of $G$ such that $g_{n}^{\prime}\left(a_{i}\right)=a_{i}^{n}$. Then $g_{n} g_{n}^{\prime}\left(a_{i}\right)=A_{i}^{n} \rightarrow A_{i}$. Letting $i=1$ we see that $g_{n} g_{n}^{\prime}\left(a_{1}\right) \rightarrow A_{1}$. Since $a_{1}$ certainly approaches $a_{1}$, Theorem 6.1 tells us that the sequence $g_{n} g_{n}^{\prime}$ contains a subsequence converging to an element $g^{*}$ in $G$. We assume that our original sequence above is taken as this convergent subsequence. An application of Theorem 6.1 to the sequence $g_{n}^{\prime}$ (remembering that $g_{n}^{\prime}\left(a_{1}\right)=a_{1}^{n} \rightarrow a_{1}$ ) shows that for a subsequence of $n$ 's (which we now take for the whole sequence) $g_{n}^{\prime} \rightarrow g^{\prime}$ where $g^{\prime}$ is in $G$. We have now arrived at the following situation:

$$
g_{n} g_{n}^{\prime} \rightarrow g^{*}, \quad g_{n}^{\prime} \rightarrow g^{\prime} .
$$

By the preceding results $g_{n}^{\prime-1} g_{n}^{-1} \rightarrow g^{*-1}$. Hence 


$$
g_{n}^{\prime}\left(g_{n}^{\prime-1} g_{n}^{-1}\right)=g_{n}^{-1} \rightarrow g^{\prime} g^{*-1}, \quad g_{n} \rightarrow g^{*} g^{\prime-1} .
$$

Now for any $i(1 \leqq i \leqq k)$

$$
A_{i}^{n}=g_{n}\left(a_{i}^{n}\right) \rightarrow g^{*} g^{-1}\left(a_{i}\right) .
$$

But $A_{i}^{n} \rightarrow A_{i}$ and hence

$$
g^{*} g^{\prime-1}\left(a_{i}\right)=A_{i} .
$$

The element $g^{*} g^{\prime-1}$ therefore has the desired properties.

This theorem applied to the plane case (and with $k=3$ ) yields Hilbert's Axiom III. It is worth pointing out that we have actually proved a great deal more than Hilbert's axioms; we have also obtained many of the results of his paper. But as we are, at the moment, only interested in establishing that our weaker axioms suffice for the plane, we leave the plane case and turn our entire attention to the three dimensional case. From now on it is to be understood that we are dealing with this latter case.

13. By a G-straight, or more simply a straight or a line we shall mean a topological line which is the set of points left fixed by a circular subgroup of some $G_{x}$. Through every point of space there is clearly a large family of straights. Now let $x$ and $y$ be any two distinct points of the space. In the group $G_{x}$ there is one and only one circular subgroup leaving $y$ fixed. Let this group be called $K_{x y}$. It clearly leaves fixed a topological line, call it $L_{x y}$. We have therefore seen that there is at least one $G$-straight through every two distinct points of space. We know that $L_{x y}$ is the set of all fixed points of $K_{x y}$. We have, therefore, the following theorem.

THEOREM 7. Through each pair of distinct points of $E$ there passes one and only one G-straight.

13.1. It is worth noting that if an element $g$ in $G$ leaves fixed three points $x, y, z$ not all on the same straight then $g$ leaves all of $E$ fixed. For since $g$ leaves $x$ fixed it is in $G_{x}$. Since it leaves $y$ fixed it is in the circular subgroup $K_{x y}$ of $G_{x}$; and since it leaves fixed $z$, a point not on the "axis" of $K_{x y}$, it must leave all of $E$ fixed.

The symbol $x y$ will be used to denote the closed portion of the line $L_{x y}$ which is contained between the points $x$ and $y$. This set of points will be called an interval or the interval $x y$, or a segment.

Let $L_{x y}$ be a straight, left fixed by the circular group $K_{x y}$, and let $g$ be any element of $G$. Then the set of points $g\left(L_{x y}\right)$ is the set left fixed by $g K_{x y} g^{-1}$. In other words the image of a straight under any element of $G$ is also a straight. It follows that the image of a segment is a segment.

Two configurations of the space $E$ are said to be congruent if one of them is carried into the other by some element of $G$.

Any two straights are congruent and in fact any two marked straights are 
congruent. By a marked straight we mean a straight with some one of its points particularly "marked." It is furthermore true that any marked straight can be taken to any other so that a given direction on the one goes to a given direction on the other. These facts follow from the transitivity of $G$ and the nature of the rotation group.

14. As we have said, a sphere is defined to be any two dimensional orbit of any group $G_{x}$. The point $x$ is called the center of the sphere.

TheORem 8. A straight and a sphere can intersect in two points at most.

Let $x$ be the center of $S$ the sphere and let $L$ be the straight. Suppose $a$ and $b$ are two points of intersection of $L$ and $S$. There exists an element of $G_{x}$ which interchanges $a$ and $b$. This element must carry $L$ into itself since $L$ is determined by any two of its points. Then there exists a non-trivial subgroup of $G_{x}$ which leaves $L$ invariant. This subgroup which will be denoted by $Q$ is compact. Such a compact group acting on a line can contain only two distinct transformations, the identity and a reflection. Hence under this group the orbit of $a$ consists of the two points $a$ and $b$. It follows that there can be no other point $c$ of $L$ on $S$, for otherwise there would be an element of $Q$ interchanging $a$ and $c$ and $a$ would have at least three points in its orbit. This completes the proof.

THEOREM 9. If p and $q$ are inside a sphere $S$, then the segment $p q$ is inside $S$.

The straight $L_{p q}$ is not compact in either direction. In going along $L_{p q}$ from $p$ to $q$ and on we must meet $S$ in some point. Similarly we must meet $S$ in going from $q$ to $p$ and on out. The straight $L_{p q}$ meets $S$, then, in two points neither of which is in the interval $p q$. Therefore no point of the interval can be outside $S$ for this would imply that some point of the interval was on $S$ and this would mean that $L_{p q}$ had at least three distinct points on $S$.

15. Theorem 10. If $x_{n} \rightarrow x$ and $y_{n} \rightarrow y$ then $x_{n} y_{n} \rightarrow x y$.

Let $z_{n}, n=1,2,3, \cdots$, be a point of $x_{n} y_{n}$. Any sphere surrounding $x y$ surrounds almost all the $z_{n}$, so that for a proper subsequence of the $n$ and a suitable point $z, z_{n} \rightarrow z$. We have to prove that $z$ is on $x y$. There exist elements $g_{n}$ such that $g_{n} x_{n}=x$, and $g_{n} y_{n}$ is on $L_{x y}$ on the same side of $x$ as the point $y$.

Now we know that there is a subsequence of the $g_{n}$ and an element $g$ such that $g_{n} \rightarrow g$. Further $g x=x, g_{n} y_{n} \rightarrow g y$, and $g_{n} z_{n} \rightarrow g z$. Now $g_{n} y_{n}$ is on $L_{x y}$ so that $g y$ must also lie on $L_{x y}$. On the other hand $g y$ belongs to $G_{x}(y)$. Then it is clear that $g y=y$. Therefore $g$ belongs to $K_{x y}, g^{-1}$ belongs to $K_{x y}$ and $z=g^{-1}(g z)$ is a point of $L_{x y}$ since $g z$ is a point of $L_{x y}$. It is now a trivial matter that $z$ is on $x y$ and that every point of $x y$ is a limit point of some sequence $z_{n}$.

It should be remarked that under the same hypothesis the line $L_{x_{n} y_{n}}$ converges to the line $L_{x y}$. By this we mean that every sequence $z_{n}$ of points from 
$L_{x_{n} y_{n}}$ either has no limit point or every limit point which it has is on $L_{x y}$. Furthermore every point of $L_{x y}$ is a limit point of such a sequence.

Let us now take a point $z_{n}, n=1,2,3, \cdots$, on $L_{x_{n} y_{n}}$ and assume that the sequence $z_{n}$ converges to a point $z$. The intervals $x_{n} z_{n}$ then converge to $x z$ and $y_{n} z_{n}$ converge to $y z$. It may be assumed that the points $z_{n}$ are outside the interval $x_{n} y_{n}$ say in the order $x_{n} y_{n} z_{n}$. The segment $x z$ then contains $x y$ and $y z$, so that $x z$ is clearly part of the. line $L_{x y}$.

The argument that every point of $L_{x y}$ is such a limit is not difficult.

16. TheOREM 11. Let $x$ and $y$ be any two points of the sphere S. Then the interval $x y$, except for $x$ and $y$, is inside $S$.

Let $x_{n}$ and $y_{n}$ be sequences of points inside $S$ converging respectively to $x$ and $y$. By a preceding theorem the intervals $x_{n} y_{n}$ must be contained in the interior of $S$. The limit of these intervals will then be inside or on $S$. This limit is $x y$. This shows that $x y$ is entirely contained in $S$ and its interior. But $L_{x y}$ can have no point besides $x$ and $y$ on $S$. The conclusion therefore follows.

17. A point $y$ is said to be the midpoint of the segment or interval $x z$ if it is on $x z$ and if there is an element of $G$ leaving $y$ fixed and interchanging $x$ and $z$. The point $y$ is then the center of a sphere containing $x$ and $z$ as antipodal points.

THEOREM 12. Every segment has a unique midpoint.

We begin by showing the existence of the midpoint. Let $y$ be a variable point of the segment $x z$. There is in $G_{y}$ an element of order two which moves $x$ to a unique point of $L_{x z}$. Let this unique point be denoted by $f(y)$. Now $f(x)=x$; and $f(z)$ lies on $L_{x y}$ and has the order $x z f(z)$. Therefore if $f(y)$ is continuous it will assume for some $y$ the value $z$. We have only to show therefore that $f(y)$ is continuous.

Let $y_{n}$ approach $y_{0}$. Lemma 6 shows that $f\left(y_{n}\right)$ is a bounded set, and we may assume that $f\left(y_{n}\right)$ approaches a point $w$ of the line $L_{x z}$. We wish to show that $w=f\left(y_{0}\right)$. Assume that this is not true and that $w$ is distinct from $f\left(y_{0}\right)$. The pair $\left(x, y_{n}\right)$ is carried by an element of the group to the pair $\left[f\left(y_{n}\right), y_{n}\right]$. There must be an element $g$ of the group taking the pair $\left(x, y_{0}\right)$ to the pair $\left(w, y_{0}\right)$. There is also an element $g^{\prime}$ taking the pair $\left(x, y_{0}\right)$ to the pair $\left(f\left(y_{0}\right), y_{0}\right)$. That is

$$
g\left(y_{0}\right)=g^{\prime}\left(y_{0}\right)=y_{0}
$$

and

$$
g(x)=w, \quad g^{\prime}(x)=f\left(y_{0}\right) .
$$

Hence $g^{\prime} g^{-1}$ leaves $y_{0}$ fixed and takes $w$ to $f\left(y_{0}\right)$. This is impossible because $g^{\prime} g^{-1}$ is in the compact group $G_{y_{0}}$ and the points $w$ and $f\left(y_{0}\right)$ are both on the 
same side of $y_{0}$ on the line $L_{x z}$. Hence $f(y)$ is continuous and the midpoint of $x z$ exists.

Assume now that there are two midpoints $y$ and $y^{\prime}$ of the segment $x z$. Each of them gives rise to a reflection interchanging $x$ and $z$ and leaving itself fixed. The product of these two reflections of $L_{x z}$ is a transformation of $L_{x z}$ leaving $x$ and $z$ fixed and moving other points on the line. This is impossible.

18. The next geometric concept to be defined will be the projection of a point $z$ on a line $L$. If $z$ is on $L$ this projection is defined to be $z$ itself. If $z$ is not on $L$ let $g$ be an element of order two in $K_{L}$ which is the circular group leaving every point of $L$ fixed. Let $z^{\prime}=g(z)$; evidently $g\left(z^{\prime}\right)=z$. The line $L_{z z^{\prime}}$ and the segment $z z^{\prime}$ are invariant under $g$, so that $g$ is a reflection of the line and segment with a fixed point $p$. Since this point $p$ is fixed under an element of $K$ not the identity, it is fixed under all of $K$ and is on $L$. The point $p$ is now defined to be the projection of $z$ on $L$.

THEOREM 13. The projection of $z$ on $L$ is a continuous function of $z$.

Suppose $z_{n}$ converges to $z$. Then $g\left(z_{n}\right)=z_{n}^{\prime}$ converges to a point $z^{\prime}, g$ being the element of order two in the group leaving $L$ fixed. The segments $z_{n} z_{n}^{\prime}$ converge to the segment $z z^{\prime}$. The points $p_{n}$ have some limit point on $z z^{\prime}$. But a limit point of the $p_{n}$ 's must be fixed under $g$ and can only be the point $p$.

19. The space $E$ is given to us as a metric space. Our purpose now is to introduce a new metric equivalent to the old which will be invariant under $G$.

Let $L$ be any straight in $E$ and let $G^{*}$ be the set of elements of $G$ which transform $L$ into itself while preserving direction. Any element of $G^{*}$ which leaves a point of $L$ fixed leaves every point of $L$ fixed. Let $g_{n}$ be a sequence of elements of $G^{*}$ such that for some point $a$ in $L$ the sequence $g_{n}(a)$ approaches $a$. Then for any $b$ in $L$ the sequence $g_{n}(b)$ approaches $b$. It follows from the fact mentioned here that if two homeomorphisms of $G^{*}$ act approximately the same way on a single point of space then they act approximately the same way over any bounded part of the line.

It will be useful to note also that if $a b$ is an interval of $L$ and $g$ takes $L$ into itself with direction reversed and if $g(b)=a$ it follows that $g(a)=b$. With the aid of $G^{*}$ we shall now see how $L$ becomes the carrying space of a topological group.

19.1. Let $o$ be a point of $L$ fixed but arbitrary. Let $a$ and $b$ be any two points of $L$. Let $f$ be an element of $G^{*}$ which moves $o$ to $a$, and let $g$ be an element of $G^{*}$ which moves $o$ to $b$. Then by definition $a \cdot b=f g(o)$. This definition of multiplication on $L$ is associative. It has an inverse, and hence all the group axioms are satisfied. If $f(o)=a$, the inverse of $a$ is $f^{-1}(o)$. Another way to obtain $a^{-1}$ is to define it as the position to which $a$ goes by an element which reverses direction on $L$ while leaving $o$ fixed. The latter definition shows that the operator "inverse" is continuous.

Now assume that $a_{n}$ approaches $a$ and $b_{n}$ approaches $b$. Let $f_{n}(o)=a_{n}$, 
$g_{n}(o)=b_{n}, f(o)=a$, and $g(o)=b$. By the remarks in $\$ 19 f_{n}$ is near $f$ for large $n$ and any bounded portion of the line. A similar statement may be made about $g_{n}$ and $g$. Therefore $f_{n} g_{n}(o)$ is near $f g(o)$. This shows that the group multiplication $a \cdot b$ is simultaneously continuous in $a$ and $b$.

Thus with multiplication as above defined $L$ becomes the carrier of a topological group. But it is known that such a group must be bicontinuously isomorphic to the additive group of real numbers. The line $L$ may now be metrized with the metric of the real numbers carried by it in this way. That is, if $x$ and $y$ are any two points of $L$, then $d^{*}(x, y)$, the new distance, is defined to be the absolute value of the difference of the real numbers corresponding to $x$ and $y$.

Now suppose that the segment $x y$ is translated to $x^{\prime} y^{\prime}$ by an element $g$ of $G^{*}$. Thus:

$$
g(x)=x^{\prime}, \quad g(y)=y^{\prime} .
$$

If $g(o)=z$, then

$$
z \cdot x=x^{\prime}, \quad z \cdot y=y^{\prime} .
$$

Therefore $x \cdot y$ is translated to $x^{\prime} \cdot y^{\prime}$ by an operation of the topological group defined above and therefore $d^{*}(x, y)=d^{*}\left(x^{\prime}, y^{\prime}\right)$.

Any element of $G$ which reverses sense on $L$ also preserves the new distance. This is because there is one sense reversing transformation which merely changes the ends of a given interval. This transformation leaves the length of the interval invariant. Any other sense reversing transformation is the product of this one and an element of $G^{*}$, from which our statement now follows.

19.2. We may now define the new distance for any two points $x$ and $y$ of space. There is some element in $G$ which carries $x$ and $y$ to two points $x^{\prime}$ and $y^{\prime}$ of $L$. The distance $d^{*}(x, y)$ is defined as equal to $d^{*}\left(x^{\prime}, y^{\prime}\right)$. By its very definition this new distance extended as it now is to all of $E$ is invariant un$\operatorname{der} G$. In fact any two pairs of points are congruent if and only if they have the same distance $d^{*}$.

19.3. To show that the new distance $d^{*}$ is equivalent to the old it is only necessary to show that it is a continuous function.

Theorem 14. The distance $d^{*}(x, y)$ is a continuous function of $x$ and $y$.

Let $x_{n}$ 's converge to $x$ and $y_{n}$ 's to $y$. We saw in the proof of Theorem 10 that $G$ contains an element which takes $x_{n}$ to $x$ and $y_{n}$ to the line $L_{x y}$, near $y$. But on $L$ and hence on $L_{x y}, d^{*}$ is a continuous function. This shows that $d^{*}$ of the transformed pair, which is the same as $d^{*}$ for the original pair $\left(x_{n}, y_{n}\right)$, is near $d^{*}(x, y)$.

19.4. ThEOREM 15. The distance $d^{*}(x, y)$ satisfies the triangle axiom. 
Let $A, B$, and $C$ be any three points of $E$. We wish to prove that

$$
d^{*}(A, C)+d^{*}(C, B) \geqq d^{*}(A, B) .
$$

If $C$ is on the line $L_{A B}$ we know this. When $C$ is between $A$ and $B$ the equality holds, and this will be the only case of equality.

Suppose now that $C$ is not on $L_{A B}$ and let $C^{\prime}$ be the projection of $C$ on the line. To prove the desired inequality it will suffice to show that $d^{*}(A, C)$ $>d^{*}\left(A, C^{\prime}\right)$ and $d^{*}(C, B)>d^{*}\left(C^{\prime}, B\right)$. This follows from the following lemma.

Lemma 7. Let $x, y$, and $z$, not on a line, be such that $z$ is the projection of $y$ on $L_{x z}$. Then $d^{*}(x, y)>d^{*}(x, z)$.

Let $S$ be the sphere about $x$ which goes through $y$, that is, the sphere with center $x$ and radius $d^{*}(x, y)$. Let $g$ be the element of order two in $K_{x z}$ and let $y^{\prime}=g(y)$. The segment $y y^{\prime}$ has $z$ as midpoint, so that certainly $z$ is on $y y^{\prime}$. The point $y^{\prime}$ is on $S$ because $x y$ and $x y^{\prime}$ are congruent under $g$. If now $d^{*}(x, z)=d^{*}(x, y)$, there would have to be a point $z^{\prime}$ on the segment $x z$ such that $d^{*}\left(x, z^{\prime}\right)=d^{*}(x, y)$. Since $y$ and $y^{\prime}$ are on the sphere, $z$ is inside it. Of course $x$ is inside the sphere and hence $z^{\prime}$ is also inside it, contrary to the preceding equality.

It should be noted that all points of $y y^{\prime}$ except $y$ and $y^{\prime}$ are inside $S$ so that for any point $w$ of $y y^{\prime}$ distinct from $y$ and $y^{\prime}, d^{*}(x, w)<d^{*}(x, y)$.

It is clear that we have established the metric characterization of a straight line: three points are on a straight line if and only if their distances in proper and unique order satisfy the triangle equality. From this point on, the distance $d^{*}$, now called $d$, will be the only distance used.

20. Let $L$ and $L^{\prime}$ be two lines having in common the single point $p$ and such that there exists an element of order two in $K_{L}$ under which the line $L^{\prime}$ is invariant. In this case $L^{\prime}$ is said to be orthogonal or perpendicular to $L$. If $x$ is a point on $L^{\prime}$, its projection on $L$ is the point $p$.

Consider the rotation group $G_{p}$ and the coordinate system that goes with it which makes $G_{p}$ the three-space rotation group. Since there is an element of order two, that is, a half-rotation in $G_{p}$ which leaves $L$ fixed and $L^{\prime}$ invariant, there is also a half-rotation in $G_{p}$ which leaves $L^{\prime}$ fixed and $L$ invariant. In other words $L$ is also orthogonal to $L^{\prime}$ and the relation of orthogonality is symmetric. It can be seen also that orthogonality is a group invariant.

20.1. The transitivity of $G$ and the nature of the rotation group enables us to state the following two theorems.

Theorem 16. Let $L$ and $N$ be two lines both orthogonal to a line $M$ at a point $p$. There is then an element of $K_{M}$ which carries $L$ to $N$.

This makes it clear incidentally that the locus of points on lines orthogonal to $L$ at $p$ is a topological plane. 
THEOREM 17. Let $L$ and $L^{\prime}$ be orthogonal at $p$ and let $M$ and $M^{\prime}$ be orthogonal at $q$. There is then an element $g$ in $G$ such that $g(p)=q, g(L)=M$ and $g\left(L^{\prime}\right)=M^{\prime}$. Furthermore this element may be so chosen as to take any desired directions on $L$ and $M$ to any desired directions on $L^{\prime}$ and $M^{\prime}$.

20.2. Let $L$ and $L^{\prime}$ be a pair of orthogonal lines intersecting in a point $p$. Let $x$ and $y$ be two points of $L^{\prime}$ on opposite sides of $p$ and at equal distances from $p$. A half-rotation about $L$ must then interchange $x$ and $y$. If $q$ is any point of $L$, the half-rotation carries the segment $q x$ to $q y$, so that $q$ is equidistant from $x$ and $y$. We may express this result by saying that if two lines are orthogonal any point on one of them is equidistant from any pair of symmetrically placed points on the other.

20.3. A triangle is defined in the natural way as the system of three segments joining pairs of a set of three points. Other simple geometric concepts will sometimes be used without definition when the definition is perfectly straightforward.

As a sort of converse to the result of the preceding section the following theorem is given at this point.

THEOREM 18. The altitude of an isosceles triangle bisects the base.

Let $q, x$, and $y$ be three points such that $q x$ and $q y$ are congruent. Let $p$ denote the projection of $q$ on $L_{x y}$. Then $L_{p q}$ is orthogonal to $L_{x y}$. Consider the half rotation which leaves $L_{p q}$ fixed and $L_{x y}$ invariant. This carries $x$ and $y$ to $x^{\prime}$ and $y^{\prime}$, all four points being on the same line. All four segments $q x, q y, q x^{\prime}$, and $q y^{\prime}$ are equal and the line $L_{x y}$ must meet the sphere $G_{q}(x)$ in four points, $x, y, x^{\prime}$, and $y^{\prime}$. At most two of these are distinct and we know that $x$ and $y$ are distinct. This makes it clear that $y^{\prime}$ is $x$ and $x^{\prime}$ is $y$, so that $p$ is the midpoint of the segment $x y$.

21. Let $L$ denote a line and $p$ a point on it. Let $\pi$ denote the set of all points of space whose projection on $L$ is the point $p$. Such a set, by definition, is a plane of our geometry. It is clear that any line $M$ orthogonal to $L$ at the point $p$ belongs to $\pi$, and that every point of $\pi$ is on one such line. Now let $x$ and $x^{\prime}$ be a pair of points symmetrically situated about $p$ on the line $L$. Then all points of $\pi$ are equidistant from $x$ and $x^{\prime}$ : conversely any point equidistant from these must lie on $\pi$. Our planes may therefore be characterized as the locus of points equidistant from some pair of points. If we consider the circle group which leaves fixed the line $L=L_{x x^{\prime}}$, we see that every line $M$, as above, is generated by this group from any arbitrary one. It follows at once that our planes have the topological structure which they should.

21.1. To show that our planes are linear sets we shall borrow from Kerékjártó the notion of introducing a simple antipodal transformation $\alpha$, $n o t$ an element of $G$, defined on the whole space as follows. Under $\alpha$ the point $p$ is fixed : a point $q$ goes to that point $q^{\prime}$ on the line $p q$ which is symmetrically 
disposed about $p$. It is obvious that the straight lines through $p$, and only these, are invariant under $\alpha$.

Our metric is invariant under this transformation $\alpha$. To see this, following Kerékjártó, we need merely show that, for an arbitrary pair of points, $\alpha$ coincides with an appropriate element of $G$. To this end, let $q$ and $s$ be two points, distinct from $p$, and let $L^{\prime}$ denote a line orthogonal to $p q$ and $p s$. Let $g$ denote a half-turn about $L^{\prime}$. It is clear that $q$ and $s$ have the same images under this half-turn as under the antipodal transformation $\alpha$. It now follows immediately from the invariance of our metric that $\alpha$ carries straight lines of space to straight lines, for these as we have seen are metrically characterized.

21.2. Consider now the line $L$ and the plane $\pi$ orthogonal to it at the point $p$. Perform upon space the antipodal transformation $\alpha$ followed by a halfturn about $L$. It is clear that the points of $\pi$ are fixed points under this product-transformation. But it is important to observe that they are the only fixed points: this follows from the fact that all lines through $p$ are invariant under $\alpha$ and only those remain invariant under the half-turn which are orthogonal to $L$ at $p$. Now take two points, $q$ and $s$, in $\pi$. The line through these must be invariant because our composite transformation carries it to a line through $q$ and $s$, since these are fixed points. This transformation, moreover, preserves distances. Since it has a pair of fixed points it must leave all of the line fixed. Therefore the line through $q$ and $s$ must lie in $\pi$, the locus of fixed points. Then we have shown that with every pair of its points our plane contains the line determined by these points.

22. As we know, the plane $\pi$ is invariant under the circle group $K_{L}$. We want to show next that associated with every point $q$ of $\pi$ there is a similar circle group leaving $\pi$ invariant. Such a step will enable us to see that there is nothing special about the point $p$ and to conclude that $\pi$ is either a euclidean or hyperbolic plane under the subgroup $G^{\pi}$ of $G$ which leaves $\pi$ invariant. This group is transitive on $\pi$.

22.1. Let us introduce the notion of the projection of a point $x$ on the plane $\pi$. This is defined as that point $x^{\prime}$ of $\pi$ which is nearest to $x$. In order to see that $x^{\prime}$ is determined, let $z$ denote a point of $\pi$ and let $S$ denote a sphere with center at $x$ and radius $x z$. The solid sphere intersects $\pi$ in a compact set. For any point of $\pi$ not in this intersection the distance to $x$ must exceed $x z$. On the compact set there certainly is one "nearest" point, but conceivably more than one. Now there can be at most one such nearest point. For suppose $x^{\prime}$ and $x^{\prime \prime}$ are two points of $\pi$ at the same distance from $x$. Then the midpoint of $x^{\prime} x^{\prime \prime}$ is in $\pi$ and is nearer to $x$ than $x^{\prime}$ and $x^{\prime \prime}$ are. It is clear from the continuity of distance and the uniqueness of projection that this projection operation is continuous. We shall use this continuity in the following theorem.

THEOREM 19. Every point $q$ of $\pi$ is the projection of at least one point $q^{\prime}$ not on $\pi$. 
Consider a sphere about $P$ large enough to have the point $q$ inside of it. This sphere meets $\pi$ in a circle, call it $C$. Let $S$ denote one of the hemispheres associated with $C$. Let $S^{*}$ denote the set of projections of $S$. Since $C$ can be deformed on $S$ to a point of $S$, it can be deformed on $S^{*}$ to a point of $S^{*}$. During this deformation it must meet $q$ since $q$ is in the domain bounded by $C$. This means that $q$ is a point of $S^{*}$ as was to be shown.

22.2. Let $q^{\prime}$ be a point not on $\pi$ and let $q$, distinct from $p$, be its protection on $\pi$. Let $\pi^{\prime}$ denote the plane of lines orthogonal to $q q^{\prime}$ at $q$. This plane contains all straight lines of which it contains a pair of points, by 21.2.

Theorem 20. The planes $\pi$ and $\pi^{\prime}$ are identical.

It will first be shown that $\pi^{\prime}$ includes $\pi$. Consider the line $L_{p q}$. This line is in both planes. The coordinate system around $P$ shows us that there is one and only one line in $\pi$ which goes through $p$ and is orthogonal to $L_{p q}$. Let $L^{\prime}$ denote any line, distinct from this one, through $p$ and in $\pi$. Let $s$ denote the projection of $q$ on the line $L^{\prime}$. The point $s$ is distinct from $p$ by our choice of $L^{\prime}$. Since $s$ and $q$ are points of $\pi, L_{s q}$ belongs to $\pi$. Since it is a line of $\pi$ which goes through $q$ it is also in $\pi^{\prime}$. Therefore $s$ and $p$ are both points of $\pi^{\prime}$ and $L_{p s}$ belongs to $\pi^{\prime}$. The set of points on such lines is dense in $\pi$, and the closure of this set, which is $\pi$, must also belong to $\pi^{\prime}$.

We will now show that $\pi$ contains $\pi^{\prime}$. The plane $\pi$ has the property that each of its points is interior to a two-cell, and it must therefore be an open set in $\pi^{\prime}$. On the other hand it is a closed subset of space and is therefore closed in $\pi^{\prime}$. Then it must coincide with $\pi^{\prime}$.

23. Let $(\pi, p)$ denote a marked plane, that is to say a plane $\pi$ with some one of its points $p$ particularly specified.

Theorem 21. Any two marked planes are congruent.

Let $(\pi, p)$ and $(\sigma, s)$ be the two marked planes. Let $L$ denote a line orthogonal to $\pi$ at $p$. Such a line shall be by definition a line through $p$ orthogonal to every line of $\pi$ through $L$. This orthogonal line always exists and is unique, for it is the locus of points $p^{\prime}$ which project on $p$ when they are projected on $\pi$. This we will see as follows. Let $L^{*}$ be the totality of points projecting on $p$. As we know $L^{*}$ must contain at least one point $p^{\prime}$ distinct from $p$. Hence from previous considerations $L^{*}$ must be the line $L_{p p^{\prime}}$.

Now let $L^{\prime}$ denote a line orthogonal to $\sigma$ at $s$. The marked line $(L, p)$ may be carried to the marked line $\left(L^{\prime}, s\right)$. Since these lines completely determine $\pi$ and $\sigma,(\pi, p)$ must go into $(\sigma, s)$ by the element which takes $(L, p)$ to $\left(L^{\prime}, s\right)$. If we carry the line $L^{\prime}$ into itself by any half turn about the point $s$, the plane $\sigma$ must go into itself with orientation reversed. The marked planes are therefore congruent with a matching of any orientations that we choose on them.

24. The geometric concepts have now been analyzed sufficiently for us to 
be able to see that they satisfy the axioms usually given for a geometry, with the exception of the parallel axiom. Axioms I, II, III, and V as given by Hilbert [4] for example are all satisfied.

It is clear that there are two possible geometries satisfying our axioms for the space case, the euclidean and the hyperbolic. We now sketch rapidly one method for seeing that there are not more than two.

Let $\pi$ be any plane in $E$. The subgroup of $G$ which takes $\pi$ into itself and preserves orientation on $\pi$ can be seen to satisfy the axioms of Hilbert's paper [5]. The plane is therefore either euclidean or hyperbolic.

Since all planes are congruent to any given plane, we see that either every plane is hyperbolic or every plane is euclidean. We wish to show that the geometry induced by $G$ is either euclidean or hyperbolic according to the character of the planes.

Let $(E, G)$ and $\left(E^{\prime}, G^{\prime}\right)$ be two systems satisfying all the axioms for the space case and assume that in the two systems planes are of the same character.

Let $(\pi, p)$ be a marked plane in $E$ and let $\left(\pi^{\prime}, p^{\prime}\right)$ be a marked plane in $E^{\prime}$. Let $H$ denote a congruence correspondence between these two planes. Let $L$ and $L^{\prime}$ be the unique lines of $E$ and $E^{\prime}$ which are orthogonal to $\pi$ and $\pi^{\prime}$ at $p$ and $p^{\prime}$. The unit of length gives us a unique correspondence between $L$ and $L^{\prime}$, the only choice, and it is an arbitrary one, being which half of $L$ is mapped on which of $L^{\prime}$. Assume that this choice has been made so that we have really chosen an upper and a lower half for $E$ and also for $E^{\prime}$.

We can now choose coordinates in $E$ and $E^{\prime}$ and extend $H$ by letting points with the same coordinates correspond. The correspondence $H$ as thus extended is isometric. It preserves segments, orthogonality, lines, planes, and in fact all geometric concepts. The function $H$ also associates with every $g$ in $G$ an element $g^{\prime}$ in $G^{\prime}$ and we are therefore led to the conclusion that $(E, G)$ and $\left(E^{\prime}, G^{\prime}\right)$ are equivalent provided the planar character of the two systems is the same.

\section{Appendix}

For the space case an alternative set of axioms might be chosen as follows. Let $\left(E_{3}, G\right)$ be a system consisting of a set $G$ of sense preserving homeomorphisms of $E_{3}$. Let the following axioms be satisfied.

$2.1^{\prime \prime}$. The same as $2.1^{\prime}$.

$2.2^{\prime \prime}$. There is a point $p$ such that $G_{p}$ satisfies the conditions (a), (b), and (c):

(a) $G_{p}$ is a proper subgroup of $G$.

(b) For each $x$ distinct from $p, G_{p}(x)$ contains at least three points.

(c) For a sequence of points $p_{n}$ approaching $p, G_{p}\left(p_{n}\right)$ is at least one dimensional.

$2.3^{\prime \prime}$. The same as $2.3^{\prime}$. 
We will show that these axioms imply $2.2^{\prime}$ from which it follows that they suffice for the foundation of space geometry.

Quite as in the paper we can arrive at the situation of $\$ 5$. We have then an open set $R_{1}$ with a closure $\bar{R}_{1}$, and $\bar{H}_{1}$ is a compact effective transformation group of $\bar{R}_{1}$. The orbits of $\bar{H}_{1}$ are the same as those of $G_{p}$.

Let $H_{1}^{*}$ be the component of the identity of $\bar{H}_{1}$. The set $H_{1}^{*}(x)$ has the same dimension as $\bar{H}_{1}(x)$ and $\bar{H}_{1}$ must have orbits of dimension at least one in $\bar{R}_{1}$.

Assume now that $H_{1}^{*}$ is one dimensional. Then $H_{1}{ }^{*}$ is the circle group. As $H_{1}^{*}$ leaves fixed an "axis" of points of $\bar{R}_{1}$, not every $x$ distinct from $p$ has 3 points in its orbit under $H_{1}^{*}$. Therefore $H_{1}^{*}$ does not exhaust $\bar{H}_{1}$. But $H_{1}^{*}$ divides $\bar{R}_{1}$ locally (near $p$ ) into a decomposition space which is essentially a half plane. $\bar{H}_{1}-H_{1}^{*}$ must act on this half plane and the only compact group which can act on a half plane is a group of order two which reflects its edge. Hence in any case points on the "axis" of $H_{1}^{*}$ will have orbits of at most two points under $\bar{H}_{1}$. This shows that $\bar{H}_{1}$ cannot be one dimensional. But since it is now seen to be of dimension greater than one it must also have orbits of dimension greater than one by arguments in our earlier papers. This concludes the reduction of the present system of axioms to those of this paper.

It should be remarked here that in the presence of condition (b) above it is altogether likely that condition (c) can be relaxed perhaps merely to assert, with Hilbert, that $G_{p}\left(p_{n}\right)$ is infinite. This appears to have all of the difficulty which attends the problem of showing, if it is true, that a zero dimensional topological transformation group of three-space is necessarily finite.

We might mention in conclusion that it seems to us of some interest to determine the three-space geometries through appropriate reflection groups, along the lines on which this was done for the plane by Cairns. While it is clear that suitable conditions on "reflections" of three-space could be made to yield the axioms of this paper, the characterization of the fixed points of reflection of three-space by $P$. Smith might lead to an interesting approach.

Added in proof: Kerékjártó has informed us that he has published a further paper on this same subject in the Proceedings of the Hungarian Academy of Sciences (1928) (in Hungarian). He is about to publish another paper on this subject in the Acta Mathematica.

\section{BIBLIOGRA PHY}

1. Alexandroff and Hopf, Topologie I, Berlin, 1935.

2. Cartan, La Théorie des Groupes Finis et Continus et l'Analysis Situs, Mémorial des Sciences Mathématiques, vol. 42.

3. Cairns, An axiomatic basis for plane geometry, these Transactions, vol. 35 (1933), pp. 234-244.

4. Hilbert, Grundlagen der Geometrie, 7th edition, 1930.

5. —_, Über die Grundlagen der Geometrie, Mathematische Annalen, vol. 56, pp. 381- 
422. This article is reprinted as appendix IV, pp. 178-230, in the edition of Hilbert's book referred to above.

6. Kerékjárt6, On a geometrical theory of continuous groups, II. Euclidean and hyperbolic groups of three dimensional space, Annals of Mathematics, (2), vol. 29, pp. 169-179.

7. Montgomery and Zippin, Periodic one-parameter groups in three-space, these Transactions, vol. 40 (1936), pp. 24-36.

8. - Compact abelian transformation groups, Duke Mathematical Journal, vol. 4 (1938), pp. 363-373.

9. - Non-abelian compact connected groups of three-space, American Journal of Mathematics, vol. 61 (1939), pp. 375-387.

10. - Topological transformation groups I, Annals of Mathematics, (2), vol. 41 (1940).

11. - A theorem on the rotation group of the two-sphere, Bulletin of the American Mathematical Society, vol. 46 (1940), pp. 520-521.

12. P. A. Smith, The topology of transformation groups, Bulletin of the American Mathematical Society, vol. 44 (1938), pp. 497-514.

13. Veblen and Young, Projective Geometry, vol. 2.

SMith College,

Northampton, Mass.,

Queens College,

FLUSHING, N. Y. 